\title{
Highly Purified Eicosapentaenoic Acid Improves Decreased Toe-Brachial Index in Japanese Patients with Type 2 Diabetes Mellitus
}

\author{
Hiroyuki Shimizu* \\ Department of Diabetes and Endocrinology, International University of Health and Welfare (IUHW) Hospital, Nasushiobara, Japan
}

\author{
Received: September 17, 2014; Accepted: November 27, 2014; Published: December 18, 2014 \\ *Corresponding author: Hiroyuki Shimizu, MD, PhD, Department of Diabetes and Endocrinology, International University of Health and Welfare \\ Hospital, Iguchi 537-3, Nasushiobara 329-2763, Japan, Tel: 81-287-37-2221 ext.2731; Fax: 81-287-39-3001; E-mail: hshimizu@iuhw.ac.jp
}

\begin{abstract}
Eicosapentaenoic acid (EPA), which is an important polyunsaturated fatty acid in fish oil, improves blood coagulability and prevents thrombosis. EPA is usually available for treatment of patients with obliterating arteriosclerosis in Japan, but its usefulness for microcirculation in the foot has not been established. We studied the effect of highly purified ethyl eicosapentaenoate on decreased microcirculation in the foot in Japanese patients with type 2 diabetes mellitus. Changes in ankle-brachial index (ABI), toe systolic pressure, and toe-brachial index (TBI) with administration of EPA were evaluated in outpatients. Individual comparison of mean values before and after treatment was performed by using paired Student $t$ test. Both decreased toe systolic pressure and TBI significantly improved with administration of ethyl eicosapentaenoate for 4 weeks in patients who had decreased TBI; decreased TBI was normalized in 24 of 35 big toes (68.6\%). In addition, treatment improved decreased $\mathrm{ABI}$; decreased $\mathrm{ABI}$ was normalized in 4 of 6 lower limbs (66.7\%). This study confirmed that administration of highly purified ethyl eicosapentaenoate improves peripheral microcirculation in diabetic patients with decreased TBI and ABI.
\end{abstract}

Keywords: Toe-Brachial Index (TBI); Ankle-Brachial Index (ABI); Eicosapentaenoic acid; Type 2 diabetes mellitus; Peripheral artery disease

\section{Introduction}

Disturbance of peripheral microcirculation in the lower limbs of diabetic patients is associated with skin ulcer and/ or foot gangrene [1]. A diagnosis of Peripheral Artery Disease (PAD) indicates a high risk of amputation in diabetic patients, but amputation of a lower limb worsens the quality of life of these patients. Improved peripheral microcirculation in the foot should effectively prevent the risk of amputation.

Fish oil, which is rich in n-3 Polyunsaturated Fatty Acid (PUFA), contains a property that prevents the development of atherosclerosis and thrombosis [2]. Dietary supplementation with n-3 PUFA has been reported to improve decreased AnkleBrachial Index (ABI) in patients with PAD [3]. Eicosapentaenoic acid (EPA), which is an important PUFA in fish oil, improves blood coagulability and prevents the development of thrombosis
[4]. The ratio of plasma EPA to Arachidonic Acid (AA) has been reported to be significantly decreased in patients with PAD, indicating that a reduced ratio of plasma EPA/AA may be involved in the development of peripheral arteriosclerosis in these patients [5]. Highly purified EPA has been reported to significantly reduce brachial-ankle pulse wave velocity in patients with cardiovascular risk factors [6] or Type 2 Diabetes Mellitus (T2DM) [7]. However, whether highly purified EPA improves peripheral microcirculation in the toes of diabetic patients with obliterating arteriosclerosis has not yet been investigated. The present data demonstrate a possible improvement of peripheral microcirculation with administration of highly purified ethyl eicosapentaenoate in the big toes of patients with T2DM who have a decreased blood supply in the foot.

\section{Subjects and Design}

\section{Subjects}

This study included 25 Japanese outpatients with T2DM (13 male and 12 female; age, $64.3 \pm 1.7$ years) who had a Toe-Brachial Index (TBI) of less than 0.6 and therefore were considered to have obliterating arteriosclerosis because the range of normal TBI values is greater than 0.6 [8].

EPA is well confirmed to be clinically available for the treatment of patients with obliterating arteriosclerosis in Japan. After the patients provided consent, highly purified ethyl eicosapentaenoate (Mochida Pharmaceuticals Co. Ltd., Tokyo, Japan) was prescribed at a dosage of $900 \mathrm{mg}$ twice a day (a total of $1800 \mathrm{mg} /$ day). The purity of ethyl eicosapentaenoate is greater than $98 \%$. The patients were treated with ethyl eicosapentaenoate over 4 weeks.

\section{Measurement}

ABI, Toe Systolic Pressure (TSB), and TBI were measured with the patient in a supine position on a bed by using the Forum ABI/PWV (Omron Colin Co., Ltd., Tokyo, Japan) [9]. TSB was measured in both big toes in each patient. $\mathrm{ABI}$ was calculated by dividing calf systolic blood pressure by brachial systolic blood pressure, and TBI was calculated by dividing big toe systolic 
blood pressure by brachial systolic blood pressure. TBI, TSB, and $\mathrm{ABI}$ were measured before and 4 weeks after the start of treatment with EPA, and the effects of ethyl eicosapentaenoate were evaluated by measuring TBI, TSB, and ABI.

\section{Statistical analysis}

All data are expressed as mean \pm SD. Statistical analysis was performed by using Prism Version 4.0b computer software. Individual comparison of the mean values before and after treatment was performed by using paired Student $t$ test. $\mathrm{P}<0.05$ was considered statistically significant.

\section{Results}

After treatment with ethyl eicosapentaenoate for 4 weeks, 10 patients had a significant decrease in TBI of both the right and left big toes and 15 patients had a significant decrease in TBI of only a single big toe (right or left). As shown in Table 1, body weight and glycemic control did not change as a result of treatment. Decreased TSB with decreased blood flow in the big toes of these patients significantly increased from $72.8 \mathrm{~mm} \mathrm{Hg}$ to $87.8 \mathrm{~mm} \mathrm{Hg}$, although brachial systolic and diastolic blood pressure did not change. Decreased TBI significantly improved from 0.48 to 0.61 and was normalized in 24 of 35 big toes (68.6\%). In addition, $A B I$ significantly increased from 1.09 to 1.13 . Although the ABI was less than 0.9 in 6 of 35 lower limbs (17.1\%) in the 35 patients with decreased TBI before the start of the treatment, the decreased ABI normalized in 4 of these 6 lower limbs (66.7\%).

\section{Discussion}

This study showed that administration of highly purified ethyl eicosapentaenoate for 4 weeks significantly improved decreased TSB and TBI in Japanese patients with T2DM, independently of glycemic control and blood pressure in the general circulation. ABI also improved after 4 weeks of treatment. Low TSB and TBI are important risk factors for amputation [2], so improvement in TSB and TBI with administration of highly purified ethyl eicosapentaenoate should contribute to the prevention of skin ulcer and/or gangrene in the feet of patients with T2DM who have PAD.

Table 1: Changes of metabolic parameters before and at 4 weeks after the start of high purity ethyl icosapentatenoate administration.

\begin{tabular}{|l|c|c|}
\hline & before & 4 weeks \\
\hline Body weight $(\mathrm{kg})$ & $64.3 \pm 1.7$ & $63.7 \pm 1.6$ \\
\hline Blood glucose $(\mathrm{mg} / \mathrm{dl})$ & $170.2 \pm 14.4$ & $174.1 \pm 11.3$ \\
\hline HbA1c $(\%)$ & $7.75 \pm 0.34$ & $7.59 \pm 0.31 \mathrm{~S}$ \\
\hline BP (mmHg) & $135.6 \pm 3.2$ & $138.8 \pm 2.7$ \\
\hline DBP (mmHg) & $76.8 \pm 2.3$ & $79.6 \pm 1.9$ \\
\hline TSB (mmHg) & $72.8 \pm 8.5$ & $87.8 \pm 6.1^{* *}$ \\
\hline TBI & $0.48 \pm 0.03$ & $0.61 \pm 0.02^{* *}$ \\
\hline ABI & $1.09 \pm 0.03$ & $1.13 \pm 0.02^{*}$ \\
\hline
\end{tabular}

${ }^{*} p<0.05$; ${ }^{* *} p<0.01 v s$. the value before the start of high purity EPA administration. Data represent mean \pm SD. SBP: Systolic Blood Pressure, DBP: Diastolic Blood Pressure.
The EPA/AA ratio has been reported to be significantly lower in patients with PAD [6], but administration of highly purified ethyl eicosapentaenoate significantly increases this ratio [10]. We have previously demonstrated a significant reduction of plasma thrombin-antithrombin III complex with administration of ethyl eicosapentaenoate for 4 weeks in patients with T2DM who have albuminuria [11]. The present data showed that the effect of ethyl eicosapentaenoate on peripheral circulation in the toes is apparent after only 4 weeks of treatment. Therefore, an improvement in hypercoagulability with use of highly purified ethyl eicosapentaenoate contributes to improvement of peripheral circulation in big toes. Furthermore, the Japan EPA Lipid Intervetion Study (JELIS) showed that treatment with ethyl eicosapentaenoate markedly reduced the occurrence of coronary artery disease in patients with PAD [12]. It is supposed that administration of highly purified ethyl eicosapentaenoate is beneficial in patients with T2DM who have PAD.

In conclusion, highly purified ethyl eicosapentaenoate improves peripheral microcirculation in the toes of patients with T2DM who have decreased TBI, independently of glycemic control and blood pressure. Administration of highly purified ethyl eicosapentaenoate is beneficial in preventing the development of gangrene and avoiding amputation because of gangrene in patients with T2DM who have PAD.

\section{References}

1. Carter SA, Tate RB. The value of toe pulse waves in determination of risks for limb amputation and death in patients with peripheral arterial disease and skin ulcers or gangrene. J Vasc Surg. 2001; 33(4): 708-14.

2. Kromhout D, Bosschieter EB, de Lezenne Coulander C. The inverse relation between fish assumption and 20-year mortality from coronary disease. N Engl J Med 1985; 312(19): 1205-9.

3. Carrero JJ, Lopez-Huertas E, Salmoeron LM, Baro L, Ros E. Dairy supplementation with (n-3) PUFAs, oleic acid, folic acid, and vitamin B-6 and E increases pain-free walking distance and improves risk factors in men with peripheral vascular disease.. J Nutr 2005; 135(6): 1393-9.

4. Ceriollo A, Giugliano D, Quatrano A, Marchi E, Barbanti M, Lefevre PJ. Evidence for a hyperglycemia-dependent decrease of antithrombin III-thrombin complex formation in humans. Diabetologia. 1990; 33(3): 163-7.

5. Fujihara M, Fukata M, Odashiro K, Maruyama T, Akashi K, Yokoi Y. Reduced plasma eicosapentaenoci acid-arachidonic acid ratio in peripheral artery disease. Angiology. 2013; 64(2): 112-8. doi: $10.1177 / 0003319712437031$.

6. Fukuoka Y, Nuruki N, Amiya S, Tofuku K, Aosaki S, Tsubouchi H. Effects of a fish-based diet and administration of pure eicosapentaenoic acid on brachila-ankle pulse wave velocity in patients with cardiovascular risk factors. J Cardiol. 2014 ; 63(3): 211-7. doi: 10.1016/j. jjcc.2013.08.005.

7. Mita T, Watada H, Ogihara T, Nomiyama T, Ogawa O, Kinoshita J, et al. Eicosapentaenoic acid reduces the progression of carotid intimamedia thickness in patients with type 2 diabetes. Atherosclerosis. 2007; 191(1): 162-7.

8. Carter SA. Role of pressure measurements in vascular disease. In: 
Bernstein EF, editor. Noninvasive Diagnostic Techniques in Vascular Disease. 3rd ed. St. Louis: The Mosby Incorporated; 1985. p. 513-74.

9. Shimizu H, Shimomura K, Negishi M, Oh-I S, Tomita Y, Uehara Y, Mori M. Glycaemic control and the increase in pulse wave velocity (PWV) in type 2 diabetic patients. Diabetic Med. 204; 21: 804-5.

10. Ohnishi H, Saito Y. Eicosapentaenoic acid (EPA) reduces cardiovascular events: relationship with the EPA/arachidonic acid ratio. J Atheroscler Thromb. 2013; 20(12): 861-77.
11. Shimizu H, Ohtani K, Tanaka Y, Fukatsu A, Uehara Y, Sato N, et al. Increased plasma thrombin-antithrombin III complex levels in noninsulin dependent diabetic patients with albuminuria are reduced by ethyl icosapentatenoate. Thromb Haemost. 1995; 74(5): 1231-4.

12. Ishikawa Y, Yokoyama M, Saito Y, Matsuzaki M, Origasa H, Oikawa S, et al. Preventive effects of eicosapetaenoic acid on coronary artery disease in patients with peripheral artery disease. Circ J. 2010; 74(7): 1451-7. 